\title{
Perfil das notificações de incidentes em saúde em um hospital universitário
}

Profile of health incidents notifications at a university hospital

Perfil de notificaciones de incidentes de salud en un hospital universitario

Cláudia Novais Dias'; Mônica de Almeida Carreiro"

\begin{abstract}
RESUMO
Objetivo: delinear o perfil das notificações de incidentes em saúde em um hospital universitário do Rio de Janeiro. Método: estudo retrospectivo e descritivo com abordagem quantitativa, realizado em um hospital universitário do Rio de Janeiro. Os dados foram coletados das fichas de notificação de incidentes em saúde referente ao período de outubro de 2015 a maio de 2018.Resultados: no total, foram analisadas 534 notificações de incidentes em saúde. Os enfermeiros foram os principais notificadores, com um predomínio expressivo das queixas técnicas, destacando a prevalência da tecnovigilância. Os incidentes com dano ocorreram com maior concentração nos setores de internação. Conclusão: a partir do perfil das notificações foi possível evidenciar fragilidades no sistema que sugerem a existência da subnotificação na instituição, sendo um entrave para o entendimento das ocorrências.
\end{abstract}

Descritores: Segurança do Paciente; Gestão da Segurança; Qualidade da Assistência à Saúde; Notificação.

\begin{abstract}
Objective: to profile health incident notifications at a university hospital in Rio de Janeiro. Method: in this quantitative, retrospective, descriptive study at a university hospital in Rio de Janeiro, data were collected from health incident notification sheets for October 2015 to May 2018. Results: 534 health incident notifications were examined. Nurses were the main notifiers, and there was a significant prevalence of technical complaints, predominantly technovigilance-related. Incidents involving damage were concentrated more in inpatient sectors. Conclusion: the profile of notifications made it possible to highlight weaknesses in the system that suggest the existence of underreporting in the institution, which is an obstacle to understanding the occurrences.
\end{abstract}

Descriptors: Patient Safety; Safety Management; Quality of Health Care; Notification.

\section{RESUMEN}

Objetivo: perfilar las notificaciones de incidentes de salud en un hospital universitario de Río de Janeiro. Método: en este estudio cuantitativo, retrospectivo y descriptivo en un hospital universitario de Río de Janeiro, se recolectaron datos de las hojas de notificación de incidentes de salud de octubre de 2015 a mayo de 2018. Resultados: se examinaron 534 notificaciones de incidentes de salud. Las enfermeras fueron los principales notificadores y hubo una prevalencia significativa de quejas técnicas, predominantemente relacionadas con la tecnovigilancia. Los incidentes con daños se concentraron más en los sectores de hospitalización. Conclusión: el perfil de notificaciones permitió resaltar debilidades en el sistema que sugieren la existencia de subregistro en la institución, lo cual es un obstáculo para el entendimiento de las ocurrencias.

Descriptores: Seguridad del Paciente; Administración de la Seguridade; Calidad de la Atención de Salud; Notificación.

\section{INTRODUÇÃO}

Os avanços ocorridos na área da assistência à saúde e no contexto hospitalar, tornaram o cenário da saúde mais complexo, agregando diversos elementos, avanços tecnológicos, envelhecimento populacional e gerando um aumento nas demandas e nos custos. Essas modificações trouxeram novos desafios para o setor face ao maior potencial de risco e vulnerabilidade desses sistemas'.

Neste panorama, o movimento mundial pela segurança do paciente surgiu a partir de uma grande preocupação em diferentes sistemas de saúde pelo mundo com a amplitude da ocorrência de eventos danosos relacionados à assistência no ambiente hospitalar, impactando na vida de indivíduos e sociedade ${ }^{2}$.

Neste contexto, os eventos danosos são denominados de eventos adversos (EA) e estão relacionados ao aparecimento de um problema de saúde proveniente do cuidado, e não pela doença primária ou de base, causando uma lesão não intencional que resulta em incapacidade temporária ou permanente, prolongamento do tempo de permanência, ou até a morte em decorrência do cuidado prestado ${ }^{3}$.

'Enfermeira. Mestre. Especialista em qualidade em saúde e segurança do paciente. Universidade Federal do Estado do Rio de Janeiro, Brasil. E-mail: claudia.dias@unirio.br. ORCID: https://orcid.org/0000-0003-1948-700X

"Enfermeira. Doutora. Professora Adjunta II. Universidade de Vassouras, Brasil. E-mail: carreiroma59@gmail.com. ORCID: https://orcid.org/0000-0003-1594-6491 
Diante da magnitude destes eventos, pesquisas começaram a ser realizadas no cenário internacional e nacional. Uma investigação científica que englobou 58 hospitais localizados em diferentes países em desenvolvimento (Argentina, Colômbia, Costa Rica, México e Peru), encontrou uma prevalência de 10,5\% de eventos adversos entre os pacientes internados pesquisados, $60 \%$ considerados evitáveis, acarretando comprometimentos físicos e até mesmo óbito 4 .

No Brasil, estudo realizado em um hospital de ensino, demonstrou que os eventos adversos impactaram diretamente no tempo de permanência hospitalar e na mortalidade ${ }^{5}$. Tais eventos têm impacto expressivo na morbidade e mortalidade mundial. Cada vez mais se evidencia a evitabilidade dos eventos adversos, podendo chegar a 70,2\% a redução dos danos, por meio da prevenção com abordagens abrangentes e sistêmicas ${ }^{6}$.

Outra pesquisa em hospitais brasileiros evidenciou uma taxa de incidência de eventos adversos em 7,6\%, considerando $67 \%$ como evitáveis ${ }^{7}$, demonstrando que a ocorrência dos mesmos está intrinsecamente relacionada aos riscos existentes no ambiente de atenção à saúde. Cada vez mais evidencia-se a evitabilidade destes eventos por meio da prevenção dos riscos com estratégias abrangentes e sistêmicas ${ }^{8}$.

Diante desta conjuntura, a discussão sobre a qualidade da assistência está intrinsecamente relacionada à dimensão da segurança do paciente com enfoque na prevenção dos danos causados aos usuários dos serviços de saúde. Compreende-se como segurança do paciente a redução ao mínimo aceitável do risco de dano desnecessário associado ao cuidado de saúde 9 .

Estes danos tornaram-se objeto crescente de interesse para pesquisadores. Entender quando, como e por que esses problemas ocorrem, assim como suas consequências, tornou-se uma tarefa fundamental com vias na melhoria da qualidade do cuidado'.o destaque dado aos eventos adversos impulsionou o desenvolvimento de estratégias e propostas para a redução dos danos aos pacientes com ações focadas nos riscos existentes nas instituições de saúde ${ }^{9}$.

Prevenir os eventos adversos requer mapear os riscos existentes no cenário da assistência à saúde, identificandose todas as condições e situações que favoreçam a sua vulnerabilidade. A redução da ocorrência de incidentes danosos está relacionada às ações de monitoramento e mitigação de todos os tipos de incidentes em saúde ${ }^{10}$.

De acordo com a Classificação Internacional de Segurança do Paciente (International Classification for Patient Safet - ICPS), Incidente em Saúde pode ser definido como uma circunstância ou evento que poderia, ou resultou, em um dano desnecessário ao paciente, ou seja, situações que poderiam ter sido evitadas. Quando o dano ocorre, é chamado de evento adverso ${ }^{9}$.

Nesta perspectiva, o sistema de notificação voluntária de incidentes em saúde tornou-se um dos pilares na gestão da segurança dos cuidados, captando informações quanto aos pontos de fragilidades da instituição, que auxiliam no reconhecimento do panorama situacional ${ }^{10}$. Este sistema surgiu com o propósito de fortalecer a segurança do paciente com enfoque no aprendizado organizacional diante dos eventos, priorizando uma análise sistêmica do incidente, minimizando o dano e proporcionando o entendimento das situações de risco'.

Um sistema de notificação eficiente é capaz de capturar eventos de diferentes naturezas e gravidades. Apesar da busca pela prevenção de eventos adversos, todos os incidentes são considerados primordiais para o mapeamento das situações de risco, até mesmo aqueles que não atingiram o paciente, devendo haver um estímulo ao seu relato ${ }^{11}$.

Entretanto, a subnotificação ainda é uma barreira a ser vencida por estes sistemas, uma vez que subestima o quantitativo real dos incidentes na instituição, impedindo a identificação do cenário real, podendo estar relacionada à abordagem punitiva do erro, desconhecimento sobre a temática ou sobre como notificar, sobrecarga de trabalho, entre outros $^{12}$

A busca por uma assistência segura deve ser um compromisso de todos que atuam nas organizações de saúde. Para tanto, é imprescindível uma cultura de segurança organizacional que incentive o relato voluntário de incidentes, baseado na transparência, confiança mútua e aprendizado, oportunizando intervenções'.

A comunicação de incidentes em saúde é de extrema relevância para a segurança do paciente e para a melhoria da qualidade em serviços de saúde. Permite reconhecer o contexto organizacional, antes de qualquer planejamento, identificando suas deficiências e facilitando a elaboração de um programa de intervenção.

Portanto, este estudo tem como objetivo delinear o perfil das notificações de incidentes em saúde realizadas em um hospital universitário do Rio de Janeiro.

\section{MÉTODO}

Trata-se de um estudo retrospectivo e descritivo com abordagem quantitativa, realizado em um hospital universitário situado no município do Rio de Janeiro. Os dados foram coletados pela autora principal durante a fase de análise de todas as fichas de notificação voluntária de incidentes em saúde encaminhadas ao Núcleo de Segurança do Paciente (NSP) no período de outubro de 2015 a maio de 2018. No período estudado as notificações eram realizadas 
em um formulário impresso, único, com campos em aberto, de livre preenchimento que eram distribuídos pelos setores. As fichas eram depositadas em uma urna localizada na supervisão de enfermagem ou devolvida diretamente ao NSP.

As variáveis avaliadas foram: ano da notificação, categoria do profissional notificador, setor da ocorrência, motivo da notificação e grupo do incidente (queixa técnica, circunstância de risco, "near-miss", incidente sem dano e evento adverso), e área de atuação do incidente (farmacovigilância, tecnovigilância, hemovigilância, saneantes, nutrivigilância, risco clínico/incidentes assistenciais e não clínico).

As informações coletadas foram organizadas em um banco de dados no programa Excel da Microsoft ${ }^{\circledR}$. Foi realizada análise descritiva estatística e a caracterização dos dados foi apresentada na forma de frequência observada e porcentagem.

O estudo foi aprovado pelo Comitê de Ética em Pesquisa vinculado à universidade pertencente o hospital, conforme consta no parecer consubstanciado no 2.854.111, respeitando-se todos os princípios e questões éticas da Comissão Nacional de Ética em Pesquisa (CONEP) e da Resolução nă466/12.

\section{RESULTADOS}

No total, foram analisadas e realizadas 534 notificações de incidentes em saúde referentes ao período estudado. O ano de 2017 obteve a maior concentração (56.93\%) das notificações de incidentes (tabela 1). Ressalta-se que o período de 2015 analisado foi referente à apenas o último trimestre, época contemplada com o início do sistema de notificação. Assim como, o ano de 2018 foi analisado e medido somente nos seus 5 primeiros meses. Comparando os anos de 2016 e 2017, houve um aumento de 189\% de um ano para o outro, demonstrando inicialmente uma tendência no aumento dos registros. Quando avaliada a média mensal correspondente à cada ano, houve uma variação de 3,6 à 25,3 notificações/mês. A maior média pertence ao ano de 2017, demonstrando uma ascensão do número de notificações entre os anos, até esse período. Todavia em 2018, houve um declínio dos relatos com média mensal de 11,6, estando abaixo do ano de $2016(13,4)$.

Entre os profissionais de saúde, a categoria profissional que mais notificou, de forma expressiva, foi o enfermeiro (72,47\%). O profissional médico foi uma das categorias com menor participação (0,56\%).

TABELA 1: Distribuição das notificações por ano e categoria profissional em um hospital universitário $(n=534)$, Rio de Janeiro, Brasil, 2018

\begin{tabular}{llll}
\hline & & $\mathbf{n}$ & $\mathbf{f ( \% )}$ \\
\hline \multirow{3}{*}{ Ano } & 2015 & 11 & 2,06 \\
& 2016 & 161 & 30,15 \\
& 2017 & 304 & 56,93 \\
& 2018 & 58 & 10,86 \\
& Administrativo & 16 & 3,00 \\
& Auxiliar de enfermagem & 1 & 0,19 \\
& Enfermeiro & 387 & 72,47 \\
& Fisioterapeuta & 15 & 2,81 \\
& Instrumentador cirúrgico & 1 & 0,19 \\
& Médico & 3 & 0,56 \\
& Técnico de enfermagem & 19 & 3,56 \\
& Não informado & 89 & 16,67 \\
\hline
\end{tabular}

A queixa técnica (Tabela 2 ) foi a responsável pelo maior quantitativo de relatos, representando $70.97 \%$ da amostra, seguido pela circunstância notificável (22,10\%).

A tecnovigilância foi a principal área de abrangência dos relatos, correspondendo a $74,16 \%$ das notificações. Este dado se justifica pelo principal motivo dos registros $(73,41 \%)$ estar relacionado com equipamentos $(57,68 \%)$ e artigos para saúde $(15,73 \%)$.

Neste estudo, os incidentes classificados como sem e com dano, foram todos aqueles cujas informações constantes na ficha de notificação eram suficientes para afirmar que o incidente alcançou o paciente, e se gerou dano ou não. Os incidentes comunicados que atingiram o paciente, mas que não continham informações mínimas que nos permitiam identificar algum dano foram classificados como evento sem dano. Pelo fato de nem todas as notificações fornecerem esse tipo de informação clara e definida, é possível ter havido um subdimensionamento destes eventos na 
análise. Neste estudo também não foi possível classificar o grau do dano nos eventos adversos, uma vez que não havia informações relatadas sobre os desfechos para os pacientes.

TABELA 2: Caracterização das notificações de incidentes em saúde em um hospital universitário, Rio de Janeiro, Brasil, 2018.

\begin{tabular}{llll}
\hline & & $\mathbf{n}$ & $\mathbf{f ( \% )}$ \\
\hline & Circunstância notificável & 118 & 22,10 \\
& Com dano & 20 & 3,75 \\
Grupo do incidente & Evento sentinela & 1 & 0,19 \\
& Inconsistente & 3 & 0,56 \\
& Queixa técnica & 379 & 70,97 \\
& Sem dano & 13 & 2,43 \\
\hline \multirow{5}{*}{ Área de abrangência } & Farmacovigilência & 16 & 3,00 \\
& Nutrivigilância & 1 & 0,19 \\
& Risco clínico & 21 & 3,93 \\
& Risco não clínico & 85 & 15,92 \\
& Risco ocupacional & 6 & 1,12 \\
& Saneante & 4 & 0,75 \\
& Terapia nutricional & 1 & 0,19 \\
\hline & Cadeia medicamentosa & 17 & 3,18 \\
& Artigo médico-hospitalar & 84 & 15,73 \\
& Equipamento & 308 & 57,68 \\
& Estrutura/recursos & 91 & 17,04 \\
& Processo/protocolo & 23 & 4,30 \\
& Risco biológico & 6 & 1,12 \\
& Saneante & 4 & 0,75 \\
& Terapia nutricional & 1 & 0,19 \\
\hline
\end{tabular}

Os riscos não clínicos, relacionados à fatores de infraestrutura, aparecem como a segunda área de atuação mais prevalente dentre as notificações (15,92\%), seguido do risco clínico (3,93\%). A farmacovigilância compreende apenas $3 \%$ do total dos relatos.

O percentual de incidentes relacionados ao processo assistencial (risco clínico) e à farmacovigilância foi bastante reduzido.

No que tange ao turno, $76,78 \%$ dos incidentes ocorreram na parte da manhã e $15,92 \%$ a tarde. O período diurno (7:00h às 19:00h) teve a maior concentração de eventos. A noite é responsável apenas por 3,56\%.

Os eventos adversos ocorreram com maior prevalência nos setores de internação (84,21\%), nestes setores (clínicas médicas, cirúrgicas e centros de terapia intensiva) o predomínio foi semelhante $(26,31 \%)$.

\section{DISCUSSÃO}

A inicial tendência de aumento progressivo nas notificações voluntárias observada no estudo foi relacionada ao início da implantação do NSP na instituição, a partir de 2015. O processo de estruturação se deu de forma paulatina, tanto nos aspectos gerenciais quanto estruturais, obtendo um maior alcance no desenvolvimento de ações de segurança mais sólidas, assim como composição de equipe, em 2016. Este contexto permitiu um melhor planejamento e desenvolvimento de estratégias incentivadoras da notificação e segurança do paciente neste período promovendo uma maior adesão pelos profissionais ao sistema de notificação.

Todavia, no final de 2017, iniciou um processo de mudança organizacional com redução nas atividades educacionais e campanhas de sensibilização em prol da cultura de segurança e de notificação, impactando na diminuição da comunicação dos incidentes.

A realização periódica de atividades educacionais é imprescindível para a manutenção do estímulo à notificação de incidentes, uma vez que esse tipo de estratégia, apesar de ser uma grande aliada na disseminação de conhecimento, possui uma durabilidade restrita ${ }^{12}$.

Um estudo identificou aumento de 53,89\% no número notificações voluntárias de incidentes, após a realização de intervenções educativas ${ }^{13}$. 
Considerando o período estudado nesta pesquisa, 32 meses, o quantitativo de incidentes notificados foi pequeno quando comparado à outros estudos, principalmente por se tratar de um hospital de grande porte, com nível de atenção que varia de média a alta complexidade.

Em 2015, um estudo identificou um total de 2.396 incidentes em saúde em 34 prontuários analisados de uma única clínica ${ }^{13}$. Outra investigação evidenciou um total de 2.495 incidentes no período de dois anos, também em um hospital de ensino de grande porte ${ }^{15}$.

Portanto, a subnotificação dos incidentes pode ser o motivo desta limitação, justificada pelo medo de notificar, falta de conhecimento sobre a temática e centralização das notificações em grupos de profissionais ${ }^{16}$.

A predominância do enfermeiro como notificador neste estudo é corroborada por outras pesquisas que também acharam dados semelhantes, uma atribuiu $73 \%$ dos registros de notificação à equipe de enfermagem ${ }^{12}$ e a outra identificou que apenas $0.9 \%$ das notificações foram efetuadas por médicos, demonstrando que a dificuldade da participação deste profissional favorece a subnotificação ${ }^{17}$.

Em outro hospital, os enfermeiros também lideraram as notificações de incidentes relacionados à farmacovigilância quando comparados aos médicos, e a possível justificativa para essa situação foi a insegurança no relato, medo e falta de conscientização por parte da equipe médica ${ }^{18}$.

O fato de haver o predomínio da queixa técnica foi relacionado ao fato dos profissionais se sentirem mais seguros em relatar esse tipo de notificação ${ }^{20}$. Além disto, a queixa técnica está relacionada à produtos para saúde, sem relação com o profissional, não proporcionando o sentimento de medo ou culpa ao notificar.

A prevalência da queixa técnica (81\%) também pôde ser identificada em outro estudo. Entretanto, tal investigação teve como enfoque as notificações de eventos relacionados à famacovigilância, hemovigilância e tecnovigilância. Esta situação pode justificar a predominância de queixas técnicas nesses relatos ${ }^{19}$.

O destaque da tecnovigilância nas notificações analisadas tem relação com problemas nos produtos de saúde utilizados como suporte na prática assistencial. A baixa qualidade técnica de equipamentos e insumos hospitalares foi evidenciado por alguns estudos, como um dos principais motivos para as notificações em tecnovigilância, indicando a necessidade de revisão de políticas e ações fiscalizatórias pelos órgãos competentes, assim como, revisão da modalidade de aquisição destes itens pelas instituições, considerando pré-requisitos que contemplem a qualidade ${ }^{21}$.

A criação da Comissão de Padronização de Materiais e Produtos é uma estratégia utilizada por diversas instituições que tem por finalidade criar e implementar política institucional que preze pela qualidade dos materiais adquiridos, sendo uma de suas ações a pré-qualificação de materiais médico-hospitalares, assegurando a qualidade dos produtos e, acima de tudo, da assistência ${ }^{22}$. A instituição em estudo não dispõe dessa comissão, o que pode favorecer a aquisição de produtos de qualidade ruim e não satisfatórios que comprometam a segurança de pacientes e profissionais.

A ausência de informações que permitissem uma melhor classificação do incidente, assim como o desfecho quando o incidente atingia o paciente, foi um aspecto identificado nas fichas analisadas.

Esta realidade também foi identificada em outra investigação realizada em hospital público universitário que evidenciou que $23,5 \%$ das notificações não continham a informação referente ao desfecho ao paciente, não permitindo a descrição de um resultado fidedigno para este fator. Um dos motivos atribuídos para a omissão da informação seria receio de punição da instituição por parte do profissional notificador ${ }^{23}$

As informações presentes nas notificações nem sempre são adequadas e práticas, muitas das vezes não permitindo identificar o desfecho do evento para o paciente e esta circunstância pode estar relacionada com a cultura de segurança da instituição. Quanto maior a maturidade desta cultura, melhor a qualidade dos dados informados ${ }^{24}$.

Neste estudo, a indisponibilidade de insumos e a infraestrutura desajustada, principalmente no que se refere à manutenção predial, aparecem como o principal motivo das notificações caracterizadas como riscos não clínicos.

Os riscos não clínicos são aqueles com origem em procedimentos e práticas de atividades relacionadas com a manutenção da estrutura física e dos suportes assistenciais, compreendem riscos relacionados ao ambiente'.

São inúmeros os riscos existentes no ambiente hospitalar que podem comprometer a segurança do paciente, causando danos e elevando os custos. Portanto, a identificação e avaliação do risco não clínico e desenvolvimento de proposta de ações precisam ser valorizadas'.

No que tange aos incidentes atribuídos ao processo assistencial (risco clínico) e à farmacovigilância, foram poucos os relatos identificados quando comparamos a outros estudos em instituições semelhantes. Um número muito mais expressivo (87\%) foi identificado por Bica, no percentual de eventos relacionados à assistência à saúde ${ }^{23}$. 
Incidentes relacionados à farmacovigilância apareceram como o de maior prevalência em outro cenário, sendo classificados como os que causaram mais danos ${ }^{25}$. Falhas no processo de medicação são preocupantes e surgem como a segunda principal causa do total de incidentes $(16,7 \%)^{26}$.

O reduzido quantitativo de notificações relacionadas aos processos assistenciais e farmacovigilância pode indicar uma subnotificação destes eventos na instituição estudada. Há evidências que o desconhecimento dos profissionais quanto aos erros de medicação favorece uma subnotificação ${ }^{27}$.

A subnotificação dificulta a instituição hospitalar de aumentar o conhecimento acerca da segurança dos medicamentos e outros produtos, dos processos assistenciais e organizacionais ${ }^{24}$.

A predominância dos incidentes no período da manhã e tarde é uma informação que também aparece no Sistema Nacional de Vigilância Sanitária (SNVS), demonstrando que também há o predomínio de incidentes no período diurno $(58,9 \%)$, enquanto o noturno corresponde à apenas $21,5 \%{ }^{28}$.

Outros estudos que avaliaram o turno de ocorrência dos incidentes também relataram que, nos turnos manhã e tarde, ocorreu o maior número de notificações (73,9\%), podendo ser justificado pela maior quantidade de procedimentos e exames realizados nestes períodos, principalmente pela manhã ${ }^{23}$.

A maior concentração dos eventos adversos nos setores de internação se assemelhou aos dados de outra pesquisa que identificou $64,8 \%$ das notificações provenientes de unidades de internação, devido ao maior número de pacientes nestes setores ${ }^{26}$.

A pesquisa apresentou limitações relacionadas ao sistema de notificação voluntária de incidentes, em virtude da baixa qualidade dos registros dos dados.

\section{CONCLUSÃO}

Por meio do estudo foi possível traçar o perfil das notificações realizadas na instituição identificando-se o predomínio de queixas técnicas com prevalência na área de tecnovigilância, sinalizando a existência de riscos no cenário estudado. Todavia, o reduzido número de incidentes notificados que atingem o paciente, demonstra fragilidade no sistema e indica a ocorrência da subnotificação na instituição.

Desta forma, destaca-se que incentivar o relato voluntário dos incidentes em saúde, distante de uma cultura punitiva, impulsionada pela aprendizagem e pelo compromisso de não causar dano é imprescindível para o êxito de um sistema de notificação, permitindo o conhecimento e compreensão do perfil dos incidentes, subsidiando o desenvolvimento do processo de avaliação e melhoria contínua da qualidade em saúde e segurança do paciente na instituição.

Portanto, a discussão apresentada permite refletir sobre a ocorrência de incidentes e o processo de notificação, despertando para a relevância da temática, subsidiando mudanças que sustentem a melhoria da qualidade e a segurança dos cuidados.

\section{REFERÊNCIAS}

1. Sousa P, Mendes W. Segurança do Paciente: conhecendo os riscos nas organizações de saúde. 2a ed. EaD/ENSP, Rio de Janeiro, 2019.

2. Reis CT, Martins M, Laguardia J. Patient safety as a dimension of the quality of health care: a look at the literature. Ciênc. saúde coletiva [Internet]. 2013 [cited 2019 May10]; 18(7):2029-36. DOI: https://doi.org/10.1590/S1413-81232013000700018

3. World Health Organization. The conceptual framework for the international classification for patient. Version 1.1. Genebra (Swi): WHO. 2009 [cited 2018 Aug 22]. Available from: http://www.who.int/patientsafety/en/

4. Aranaz-Andrés JM, Aibar-Remón C, Limón-Ramírez R, et al. Prevalence of adverse events in the hospitals of five Latin American countries: results of the 'Iberoamerican Study of Adverse Events' (IBEAS). BMJ Qual Saf [Internet]. 2011[cited 2019 May 10]; 20(12):1043-51. DOI: https://doi.org/10.1136/bmjqs.2011.051284

5. Roque KE, Tonini T, Melo ECP. Adverse events in the intensive care unit: impact on mortality and length of stay in a prospective study. Cad. Saúde Pública [internet]. 2016 [cited 2018 Dec 12]; 32(10). DOI: https://doi.org/10.1590/0102-311X00081815

6. World Health Organization. Summary of the Evidence on Patient Safety: implications for research. WHO, 2008 [cited 2019 Mar 5]. Available from: https://apps.who.int/iris/bitstream/handle/10665/43874/9789241596541_eng.pdf

7. Mendes W, Martins M, Rozenfeld S, Travassos C. The assessment of adverse events in Brazilian hospitals. Int. J. Qual. Health Care [Internet]. 2009 [cited 2018 Oct 12]; 21(4):279-84. DOI: https://doi.org/10.1093/intqhc/mzp022

8. World Health Organization. Summary of the Evidence on Patient Safety: implications for research. WHO. 2008 [cited 2019 Mar 5]. Available from: https://apps.who.int/iris/bitstream/handle/10665/43874/9789241596541_eng.pdf

9. World Health Organization. The conceptual framework for the international classification for patient. Version 1.1. World Health Organization (WHO). Genebra (Swi). 2009 [cited 2018 Aug 22]. Available from: http://www.who.int/patientsafety/en/ 
10. Milagres LM. Gestão de riscos para segurança do paciente: o enfermeiro e a notificação dos eventos adversos [Master thesis]. Juiz de Fora, 2015 [cited 2018 Aug 12]. Available from: http://www.ufjf.br/pgenfermagem/files/2010/05/Disserta\%C3\%A7\%C3\%A3o-Lidiane-Miranda-Milagres.pdf.

11. Agência Nacional de Vigilância Sanitária (Br). Gestão de Riscos e Investigação de Eventos Adversos Relacionados à Assistência à Saúde. Brasília, 2017 [cited 2018 Mar 22]. Available from: http://portal.anvisa.gov.br/documents/33852/Caderno+7+Gest\%C3\%A3o+de+Riscos+e+Investiga\%C3\%A7\%C3\%A3o+de+Eventos+Adversos+Relacionados+\%C3\%A0+Assist\%C3\%AAncia+\% C3\%A0+Sa\%C3\%BAde/6fa4fa91-c652-4b8b-b56e-fe466616bd57.

12. Capucho HC, Arnas ER, Cassiani SHB. Patient safety: a comparison between handwritten and computerized voluntary incident reporting. Rev. Gaúcha Enferm. [Internet]. 2013 [cited 2019 Apr 24]; 34(1):164-72. DOI: http://dx.doi.org/10.1590/S198314472013000100021

13. Capucho HC, Primo, LP. Educational interventions for stimulating voluntary reporting in a university hospital sentinel. R. Bras. Farm. Hosp. Serv. [Internet]. 2011 [cited 2019 Oct 20]; 2(2):26-30. Available from: http://www.v1.sbrafh.org.br/public/artigos/RBFHSS03_artigo_05.pdf

14. Munhoz OL, Andolhe R, Magnago TSBS, Dalmolin GL, Pasa TS. Profile of patients and incidents in a surgical clinic unit. Rev. enferm. UFPE. 2018 [cited 2018 Dec 5]; 12(2):416-23. DOI: https://doi.org/10.5205/1981-8963-v12i2a230813p416-423-2018

15. Moreira IA. Notification of adverse events: the knowledge and the role of nurses [Master thesis]. Universidade Federal de Goiás, Goiânia, 2018 [cited 2018 Dec 27]. 107f. Available from: http://repositorio.bc.ufg.br/tede/handle/tede/8759

16. Alves MFT, Carvalho DS, Albuquerue GSC. Barriers to patient safety incident reporting by healthcare workers: Integrative review. Cien. Saude Colet. [Internet]. 2017 [cited 2018 Nov 14]. http://www.cienciaesaudecoletiva.com.br/artigos/motivospara-a-nao-notificacao-de-incidentes-de-seguranca-do-paciente-por-profissionais-de-saude-revisaointegrativa/16476?id=16476.

17. Romeu GA, Távora MRF, Costa AKM, Souza MOB, Gondim APS. Notification of adverse reactions in a sentinel hospital of Fortaleza - Ceará. R. Bras. Farm. Hosp. Serv. Saúde São Paulo [Internet]. 2011 [cited 2018 Dec 27]; 2(1):5-9. Available from: http://www.sbrafh.org.br/v1/public/artigos/RBFHSS_02_art01.pdf

18. Basile LC, Santos A, Stelzer LB, Alves RC, Fontes CMB, Borgato MH, et al. Incident analysis occurrence related to potentially dangerous medicines distributed in teaching hospital. Rev. Gaúcha Enferm. [Internet]. 2019 [cited 2018 Nov 15]; 40(esp):e20180220. DOI: https://doi.org/10.1590/1983-1447.2019.20180220

19. Bezerra ALQ, Silva AEBC, Branquinho NCSS, Paranaguá TTB. Technical analysis of complaints and adverse events reported in a sentinel hospital. Rev. enferm. UERJ [Internet]. 2009 [cited 2018 Nov 15]; 17(4):467-72. Available from: http://www.revenf.bvs.br/scielo.php?script=sci_arttext\&pid=S0104-35522009000400002\&lng=pt\&nrm=iso\&tlng=pt

20. Siman AG, Cunha SGS, Brito MJM. The practice of reporting adverse events in a teaching hospital. Rev. Esc. Enferm. USP [Internet]. 2017 [cited 2018 Jun 10]; 51:e03243. DOI: https://doi.org/10.1590/s1980-220x2016045503243

21. Sousa RS, Pontes LPP, Maia JLB, Araújo HAWP, Rocha TPO, Diniz RP. Risk management in technovigilance: analysis of reports from a sentinel hospital. Rev. enferm. UERJ. [Internet]. 2017 [cited 2019 Aug 12]; 25:e22730. DOI: https://doi.org/10.12957/reuerj.2017.22730

22. Reis LB, Barbosa IA, Soares LJF, et al. Análise das reprovações de materiais médico-hospitalares de um hospital de ensino. RAHIS [Internet]. 2015 [cited 2018 Aug 27]; 12(3):10-21. DOI: https://doi.org/10.21450/rahis.v12i3.2432

23. Bica TFS, Wegner W, Gerhardt LM, Predebon CM, Neri E, Pedro R, Neri E, Breigeiron MK. Characteristics of patient safety incidents notified in a pediatric intensive care unit. Rev. enferm. UFPE [Internet]. 2017 [cited 2018 Oct 13]; 11(Supl10):4206-16. Available from: https://periodicos.ufpe.br/revistas/revistaenfermagem/article/view/231184

24. Capucho HC. Near Miss: Quase Erro ou Potencial Evento Adverso? Rev. Latino-Am. Enfermagem [Internet]. 2016 [cited 2018 Oct 13]; 19(5). Available from: http://www.revistas.usp.br/rlae/article/view/4440/5862

25. Figueiredo ML, Silva CO, Brito MFSF, D'innocenzoi M. Analysis of incidents notified in a general hospital. Rev. Bras. Enferm. [Internet]. 2018 [cited 2018 Jul 8]; 71(1):121-30. DOI: http://dx.doi.org/10.1590/0034-7167-2016-0574

26. Lorenzini E, Santi JAR, Báo ACP. Patient safety: analysis of the incidents notified in a hospital, in south of Brazil. Rev. Gaúcha Enferm. [Internet]. 2014 [cited 2019 Apr 23]; 35(2):121-7. DOI: https://doi.org/10.1590/1983-1447.2014.02.44370

27. Camerini GF, Silva DL. Patient safety: analysing intravenous medication preparation in a sentinel network hospital in Brazil. Texto Contexto - Enferm. [Internet]. 2011; 20(1):41-9 [cited 2019 Apr 23]. DOI: https://doi.org/10.1590/S010407072011000100005

28. Agência Nacional de Vigilância Sanitária (Br). Boletim Segurança do Paciente e Qualidade em Serviços de Saúde no15: Incidentes Relacionados à Assistência à Saúde - 2016 [cited 2019 Apr 23]. Brasília, 2017. Available from: http://portal.anvisa.gov.br/documents/33852/27185 5/Boletim+Seguran\%C3\%A7a+do+Paciente+e+Qualidade+em+Servi\%C3\%A7os+de+Sa\%C3\%BAde+n\%C2\%BA+15/bb6373924973-4e7f-8907-a7b3af1e297b 